\title{
Heavy Flavor Production in ATLAS
}

\author{
Thorsten Stahl \\ on behalf of the ATLAS collaboration \\ University of Siegen - Department of Physics \\ Walter-Flex-Str. 3 - 57068 Siegen - Germany
}

\begin{abstract}
ATLAS prepares a program for measurements of production cross sections both of $b$-hadrons and quarkonia in central proton-proton collisions at a new center-of-mass energy of $14 \mathrm{TeV}$ at the LHC. Dedicated triggers based on single- and di-muon signatures are designed to accommodate large statistics already in the first several months. Starting from semi-inclusive measurements at the very early stage, exclusive channels will soon dominate the measurements, allowing for tests of QCD in the Heavy Flavor sector already with $10 \mathrm{pb}^{-1}$ of data. $J / \psi$ and $\Upsilon$ polarization measurements will allow to confirm or exclude model predictions within a large interval of transverse momenta. With larger statistics, polarization measurements are being prepared for $\Lambda_{b}$. It is expected, that $30 \mathrm{fb}^{-1}$ of data collected at a luminosity of $10^{33} \mathrm{~cm}^{-2} \mathrm{~s}^{-1}$ will allow specific measurements not accessible with the statistics collected at the Tevatron. In particular, a $\Lambda_{b}$ polarization measurement will be achieved using $\Lambda_{b} \rightarrow J / \psi \Lambda$ decays.
\end{abstract}

\section{Introduction}

At the Large Hadron Collider (LHC), located at CERN, first proton-proton collisions are expected to occur this autumn. The large $b \bar{b}$-production cross section $\sigma_{b \bar{b}}$ of about $500 \mu \mathrm{barn}$ at a center-of-mass energy of $14 \mathrm{TeV}$ corresponds to $10^{3} \mathrm{~b} \bar{b}$-pairs/s at an instantaneous luminosity of $10^{31} \mathrm{~cm}^{-2} \mathrm{~s}^{-1}$. The event statistics allow for several precision measurements in the $b$-quark sector. However, the extrapolation of $\sigma_{b \bar{b}}$ from Tevatron data to LHC energies contains uncertainties of about a factor two, which requires precise measurements of total as well as of differential cross sections as functions of e.g. transverse momentum $p_{T}$ or rapidity $\eta$.

Because of the expected statistics, these measurements will cover a wide $p_{T}$ range and are suitable to improve the current precision on $b \bar{b}$-correlation measurements as well as to constrain uncertainties in NLO QCD calculations.

The prompt $J / \psi$ and $\Upsilon \rightarrow \mu^{+} \mu^{-}$cross sections measured at Tevatron show an excess compared to the theoretical description by the color singlet model (CSM). Although the color octet model (COM) describes both $d \sigma_{J / \psi} / d p_{T}$ and $d \sigma_{\Upsilon} / d p_{T}$ well, the predictions of the polarization for quarkonia decays disagree with Tevatron data $[2,3,4]$.

Dedicated trigger signatures for B-Physics have been developed in ATLAS [5], based on single- and di-muon signatures with low- $p_{T}$ thresholds. The ATLAS trigger system is a three stage system with each stage refining decisions of previous stages with higher precision. The first level (Level 1) is implemented in hardware, whereas the decisions of the High Level Trigger (HLT), consisting of Level 2 and Event Filter (EF), are computed using a large computer farm. At Level 2, a fast muon reconstruction using precision muon chambers combined with Inner Detector measurements allow for early rejection of muons from decays in flight. In order to reduce background contributions to an acceptable level, di-muon invariant mass cuts are available as well as fast vertex fitting algorithms. At the EF level, full event data as well as alignment and calibration data are used to refine the trigger decision using offline-like algorithms.

DIS 2009 


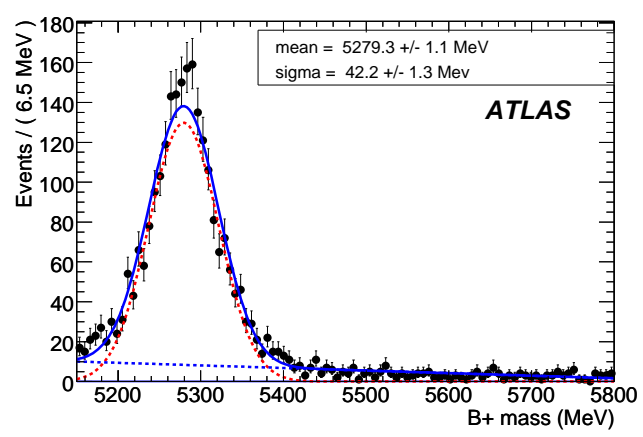

Figure 1: Invariant mass spectrum for the decay $B^{+} \rightarrow J / \psi K^{+}$(dashed red) and $b \bar{b} \rightarrow$ $J / \psi X$ background (dashed blue) contribution.

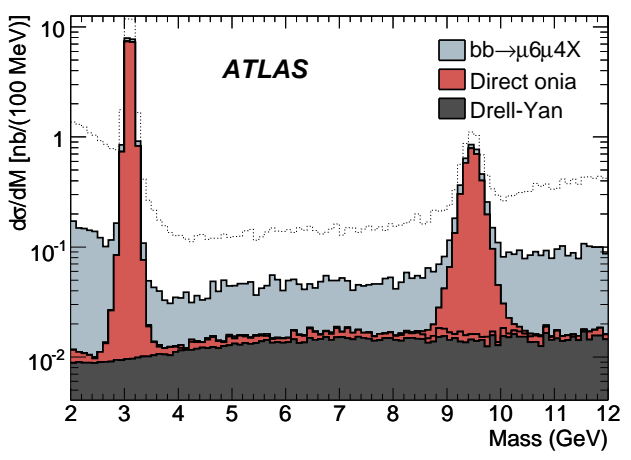

Figure 2: Invariant di-muon mass spectrum for various sources, reconstructed using a dimuon trigger. Both muons are required coming from the primary vertex. The light dotted histogram highlights the background level without vertex and pseudo-proper time cuts.

\section{Measurements of $b$ production cross sections}

For the early running period of ATLAS, inclusive as well as exclusive $b$-quark production cross section measurements are planned using heavy quarkonia, bound states of $c \bar{c}-(J / \psi)$ and $b \bar{b}-(\Upsilon)$ quarks.

\section{$2.1 \quad B^{+} \rightarrow J / \psi\left(\mu^{+} \mu^{-}\right) K^{+}$}

As an example, the exclusive channel $B^{+} \rightarrow J / \psi\left(\mu^{+} \mu^{-}\right) K^{+}$is used in ATLAS. This channel will also be utilized as a reference channel for e.g. the search for rare B-decays like $B_{s, d} \rightarrow \mu^{+} \mu^{-}$or for flavor tagging algorithm calibrations. Due to the large number of events expected in this channel, it will be used for detector performance studies like calibration and alignment of the Inner Detector as well.

The event selection is based on a di-muon trigger with muon thresholds of $p_{T}\left(\mu_{1}\right)>$ $6 \mathrm{GeV}$ and $p_{T}\left(\mu_{2}\right)>4 \mathrm{GeV}$. The offline algorithm reconstructs from two muons $\left(p_{T}\left(\mu_{1}\right)>\right.$ $6 \mathrm{GeV}$ and $\left.p_{T}\left(\mu_{2}\right)>3 \mathrm{GeV}\right)$ a $J / \psi$ with a di-muon invariant mass in a $\pm 120 \mathrm{MeV}$ wide window around the nominal $J / \psi$ mass $m_{J / \psi}^{P D G}$. The $B^{+}$meson is reconstructed by adding an additional track $\left(p_{T}>1.5 \mathrm{GeV},|\eta|<2.5\right)$, requiring an impact parameter significance of $\left|d_{0}\right| / \sigma\left(d_{0}\right)>1$ and $p_{T}\left(B^{+}\right)>10 \mathrm{GeV}$. With a total efficiency (online and offline) of $(29.8 \pm 0.8) \%$ and a di-muon trigger thresholds of $p_{T}\left(\mu_{1,2}\right)>6 \mathrm{GeV}, 4 \mathrm{GeV}$, about 1600 signal events are expected for $10 \mathrm{pb}^{-1}$ of data.

As presented in Figure 1, a mass resolution of $(42.2 \pm 1.3) \mathrm{MeV}$ is obtained. The total $B^{+} \rightarrow J / \psi K^{+}$production cross section is expected to be measured with a statistical (total) precision of $3 \%(15 \%)$, whereas the precision reached for $d \sigma / d p_{T}$ is $10 \%$ (statistical) and $16-20 \%$ (total, $p_{T}$ depending). In addition, the lifetime of the $B^{+}$meson is expected to be measured to a statistical precision of $2.5 \%$. Already with $100 \mathrm{pb}^{-1}$ of data, the statistical 
precision expected is of the order of $1 \%$. The total systematic uncertainty of the signal is about $10 \%$ and is dominated by the uncertainty in the luminosity, the uncertainty from the PDF's and the scale uncertainty of the NLO calculations.

\subsection{Heavy prompt quarkonia}

For the measurement of prompt $J / \psi$ and $\Upsilon$ production cross sections, the selection is seeded by a Level 1 di-muon trigger. In order to suppress background contributions offline (see Figure 2), both muons are required to originate from the primary vertex (vertex fit) with a pseudo-proper time of less than 0.2 ps. Employing an invariant mass window out of $m_{J / \psi}^{P D G} \pm 300 \mathrm{MeV}\left(m_{\Upsilon}^{P D G} \pm 1 \mathrm{GeV}\right)$, about $15000 \mathrm{~J} / \psi$ and $2500 \Upsilon$ are expected per $1 \mathrm{pb}^{-1}$ of data using di-muon trigger thresholds of $p_{T}\left(\mu_{1}\right)>6 \mathrm{GeV}$ and $p_{T}\left(\mu_{2}\right)>4 \mathrm{GeV}$. With a signal over background ratio of $60(J / \psi)$ and $10(\Upsilon), d \sigma / d p_{T}$ is expected to be measured to an accuracy of the order of $1 \%(J / \psi)$ and $5 \%(\Upsilon)$ for $10 \mathrm{pb}^{-1}$ of data.

Because of the large production cross section, di-muon decays of heavy quarkonia will also be used in both online and offline monitoring in ATLAS. The obtained data contains quarkonia of low $p_{T}$, complementary to $Z$ boson samples. The invariant mass $m_{\mu \mu}$ will be plotted versus a number of different variables, e.g. versus the transverse momentum, which provides checks of the muon momentum scale and the energy loss corrections. The dependence of $m_{\mu \mu}$ on the pseudorapidity can be utilized to check the correct implementation of material effects, magnetic field uniformity and stability. Detector misalignments are revealed by monitoring $m_{\mu \mu}$ as a function of the curvature $1 / p_{T}\left(\mu^{+}\right)-1 / p_{T}\left(\mu^{-}\right)$.

\section{Polarization measurements}

\subsection{Heavy prompt quarkonia}

In order to extract the polarization parameter $\alpha_{J / \psi}=\left(\sigma_{T}-2 \sigma_{L}\right) /\left(\sigma_{T}+2 \sigma_{L}\right)$, ATLAS plans to employ two different selection methods. The di-muon selection as described above has a complementary kinematic acceptance region in $\cos \theta^{*}$ compared to a selection based on a single-muon trigger, where $\theta^{*}$ specifies the polarization angle of the $\mu^{+}$w.r.t. the $J / \psi$ momentum in the rest frame of the $J / \psi$. The sensitivity is improved by combining both samples.

The single-muon selection method relies on a trigger threshold of $p_{T}(\mu)>10 \mathrm{GeV}$ and requires an additional track with $p_{T}>0.5 \mathrm{GeV}$ in a cone of $\Delta R_{\mu, \text { track }}=\left(\Delta \phi^{2}+\Delta \eta^{2}\right)^{1 / 2}<3$ around the muon direction. Both the muon and the track have to originate from the primary vertex.

With $10 \mathrm{pb}^{-1}$ of data, an estimated precision in $\alpha$ of $0.02-0.06$ and 0.2 is reached for $\alpha_{J / \psi}$ and $\alpha_{\Upsilon}$, respectively. In the case of $J / \psi$, the precision expected is comparable with Tevatron results obtained with $1.3 \mathrm{fb}^{-1}$ of data. However, ATLAS data is covering the high $p_{T}$ region, which is not accessible at Tevatron. The same competitive precision for $\Upsilon$ polarization studies will be be reached with about $100 \mathrm{pb}^{-1}$ of data.

\section{$3.2 \Lambda_{b}$ polarization}

Large polarizations have been observed in the inclusive $\Lambda$-hyperons production at energies of several hundred $\mathrm{GeV}$. Since it is still unknown if this is due to unexplained effects of existing physics or due to New Physics, $\Lambda_{b}$ polarization measurements have the possibility 
to solve the puzzle about the production mechanism of polarized $b$-quarks. With the help of the parity violating parameter $\alpha_{\Lambda_{b}}$ (see e.g. [5]), various heavy quark factorization models and pertubative QCD models will be tested.

The trigger selection for the signal channel $\Lambda_{b} \rightarrow J / \psi\left(\mu^{+} \mu^{-}\right) \Lambda\left(p \pi^{-}\right)$is seeded by a Level 1 di-muon trigger $\left(p_{T}\left(\mu_{1,2}\right)>4 \mathrm{GeV}\right)$. A $J / \psi$ meson is reconstructed offline, if the invariant di-muon mass lies within a mass window of $2.8-3.4 \mathrm{GeV}$. Both muons need to originate from the same vertex. Two tracks originating from the same vertex and with an invariant mass within $1.105-$ $1.128 \mathrm{GeV}$ (mass hypothesis assigned according to $\left.p_{T}(p)>p_{T}(\pi)\right)$ are accepted as $\Lambda$ candidates. The fraction of reconstructible $\Lambda$ for ATLAS is about $60 \%$, because the efficiency to reconstruct a $\Lambda$ decaying outside the high precision silicon layers $(r<40 \mathrm{~cm})$ vanishes due to an insufficient number of reconstructed space points. The invariant mass of the $J / \psi$ and the $\Lambda$ (both from a common vertex) has to lie in the range $5.1-6.1 \mathrm{GeV}$ with a proper transverse decay length of $d_{T}(\Lambda)>200 \mu \mathrm{m}$.

With an expected combined trigger and selection efficiency of $5.4 \%$, about 13500 $\Lambda_{b}$ events are expected for $30 \mathrm{fb}^{-1}$ of data. The $\lambda_{b}$ polarization $P$ is determined simultaneously with six parameters of helicity amplitudes from the signal decay. As presented in Figure 3, the polarization $P$ and the asymmetry parameter $\alpha_{\lambda_{b}}$ will be determined with a precision of a few percent. Their correlation varies between 0-60 \% depending on the value of $P$.

\section{Summary and conclusions}

The ATLAS collaboration is prepared to measure beauty and quarkonia production cross sections. Using the decay $B^{+} \rightarrow$ $J / \psi K^{+}$and $10 \mathrm{pb}^{-1}$ of collected data, the total cross section is expected to be measured to an accuracy of about $15 \%$, and the differential cross section $d \sigma / d p_{T}$ to $16-20 \%$. With the same amount of integrated luminosity, the precision of the $J / \psi$
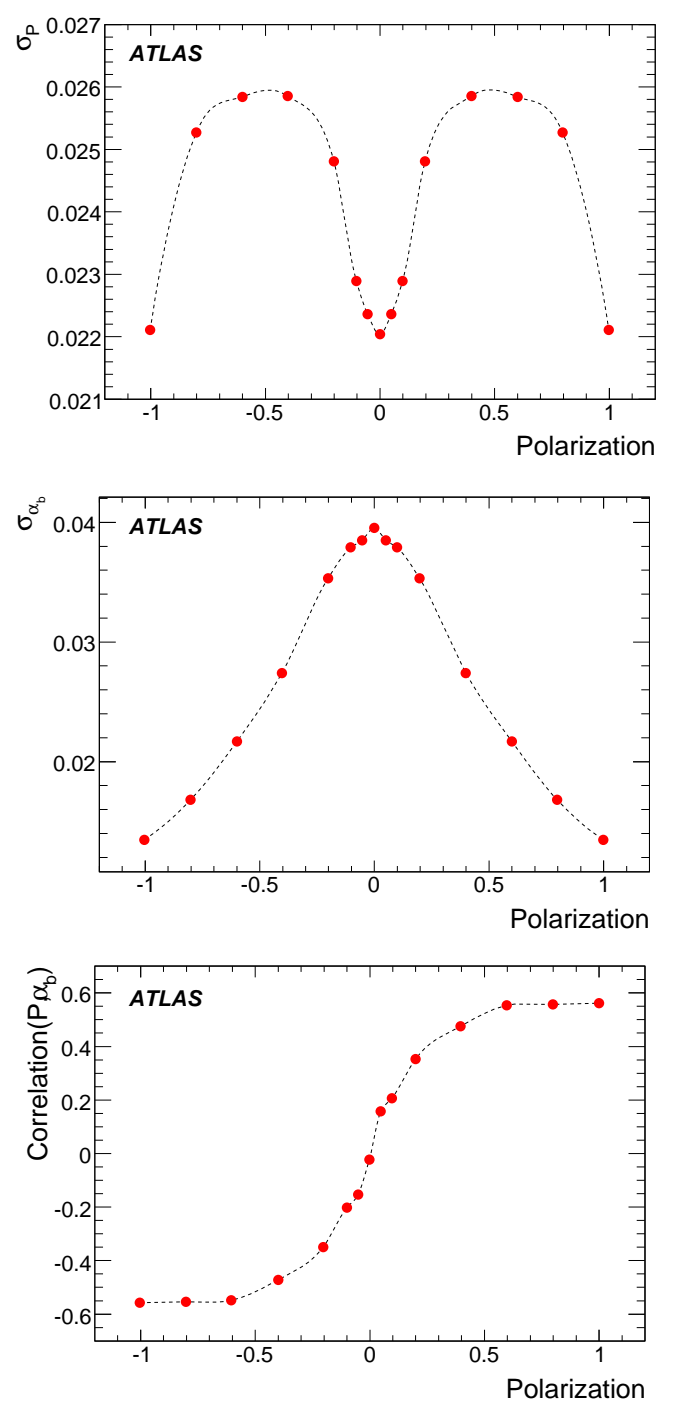

Figure 3: Expected statistical uncertainty on the polarization parameter $P$ (top), asymmetry parameter $\alpha_{\Lambda_{b}}$ (center) and the correlation of both (bottom) as a function of the polarization for $30 \mathrm{fb}^{-1}$ of data. 
polarization measurement will be comparable to the one reached with $1.3 \mathrm{fb}^{-1}$ of Tevatron data, whereas this accuracy is reached for $\Upsilon$ using $100 \mathrm{pb}^{-1}$ of ATLAS data. Both polarization measurements contain data in an interesting high $p_{T}$ region. The measurement of the expected $\Lambda_{b}$ polarization $P$ as well as the asymmetry parameter $\alpha_{\Lambda_{b}}$ determination is possible to a few percent accuracy with data of an integrated luminosity of $30 \mathrm{fb}^{-1}$. The correlation of both parameters varies between $0-60 \%$ depending on the value of the polarization.

\section{Acknowledgments}

The author is indebted to his colleagues in ATLAS, in particular S. Hassani, V. Kartvelishvili, Ch. Petridou, M. Smizanska and W. Walkowiak. He thanks the DIS 2009 conference team for this excellent conference and is grateful to the German Ministry for Education and Research (BMBF) for financial support through grant number 05HA6PS1 as part of the BMBF FSP 101 - ATLAS.

\section{References}

[1] Slides: http: //indico. cern. ch/contributionDisplay py? contribId=200\&sessionId=5\&conf Id=53294

[2] Abazov, V.M. et al. [D0 Collaboration], DØ Note 5089-conf (2007)

[3] Abe, F. et al. [CDF Collaboration], Phys. Rev. Lett. 69 (1992) 3704.

[4] Kämer, A. Prog. Part. Nucl. Phys. 47 (2001) 141

[5] Aad, G. et al. [ATLAS Collaboration], CERN-OPEN-2008-020 (2008) 